\title{
Glassy states in lattice models with many coexisting crystalline phases
}

\author{
M. J. de Oliveira ${ }^{(1)}$, A. Petri ${ }^{(2,3)}$ and T. Tomé(1) \\ (1) Instituto de Física, Universidade de São Paulo, Caixa Postal 66318, \\ 05315-970 São Paulo, São Paulo, Brazil \\ ${ }^{(2)}$ CNR, Istituto "O.M. Corbino", \\ via del Fosso del Cavaliere 100, 00133 Roma, Italy \\ (3) Unità INFM, Università La Sapienza, \\ p.le Aldo Moro 2, 00185 Roma, Italy
}

October 11, 2018

\begin{abstract}
We study the emergence of glassy states after a sudden cooling in lattice models with short range interactions and without any a priori quenched disorder. The glassy state emerges whenever the equilibrium model possesses a sufficient number of coexisting crystalline phases at low temperatures, provided the thermodynamic limit be taken before the infinite time limit. This result is obtained through simulations of the time relaxation of the standard Potts model and some exclusion models equipped with a local stochastic dynamics on a square lattice.
\end{abstract}

\section{Introduction}

Recent years have seen a growing interest in models that, albeit possessing ordered ground states, are capable of displaying glassy behaviour 1, 2, 3, 4, 5, 6. Other models contain a priori ingredients that prevent them from reaching or even possessing a crystalline state; examples of these ingredients are: quenched disorder [7], competing interactions [7, polydispersity 
[8, modified long-range potentials [9, and constrained interactions [10, 11]. While these factors may fit naturally in the description of systems characterized by a quenched disorder, like spin glasses, they seem less suitable to the description of structural glasses since they explicitly suppress crystallization, unlike most of real systems that, on the contrary, are potentially good crystal formers. The use of models with such a priori ingredients is motivated by both practical and theoretical reasons. From a practical point of view, numerical simulations are limited to relatively small lattices, so that large collective effects that in real systems could be at the origin of glassy behavior can be very depressed: e. g. molecular dynamics simulation of identical atoms interacting via a Lennard-Jones potential eventually produces crystalline configurations also in the presence of very fast cooling. From a theoretical point of view, thermodynamic stability excludes the possibility that systems with translational invariance and short range interactions could possess metastable states at finite temperatures [12, 13. On the other hand, formation of large and pure crystals is uncommon in nature, and even their growth in a laboratory is not a simple task, despite the fact that freezing of matter into non-crystalline phases is contrary to what would be expected from equilibrium thermodynamics.

For the above reasons it would be desirable to have models capable of displaying glassy behavior without the introduction of any kind of quenched disorder or special interactions, and to have simple models sheding some light on the mechanisms that drive crystal formers away from crystallization. The aim of the present letter is to show under which circumstance some simple lattice models, with neither quenched disorder nor constraining rules, are capable of relaxing into a glassy state. We will specifically investigate the Potts and the exclusion models, showing that the conditions which prevent the ordering are the same in the two models.

\section{Glassy states}

It has been observed that some exclusion models [16, 17, 18, where crystalline states are expected as a consequence of the given dynamical evolution rules, (see sections below) are pinned into some noncrystalline state. In these models the occupation of a lattice site by a particle excludes the occupation of a certain set of neighboring sites by other particles and it may happen that the effects of excluded volume prevent the system from settling down 
to the ordered state [17]. It has been found in particular [19, 20, 21] that when on a square lattice the exclusion of first and second nearest-neighbours is imposed, the system slowly approaches a state in which particles are arranged to form different macroscopic crystalline domains. However, when exclusion of third nearest-neighbours is also imposed, the system jams into noncrystalline configurations 22].

The properties of the above models suggest that the glassy state (in the sense quantitatively defined in the next sections) emerges after a sudden cooling in systems that, when in thermodynamic equilibrium, display a sufficient number of equivalent crystalline states or coexisting ordered phases at low temperatures, and a disordered phase at high temperatures. This is the case of the Potts model [23] and exclusion models [16, 18] studied here. When in equilibrium, they suffer a phase transition which is continuous if the number of ground states is small and first-order if it is large. We implement the time relaxation of these models due to the cooling process by supplying them with a local stochastic dynamics. By means of extensive numerical simulations we find that whenever the equilibrium model exhibits a continuous phase transition the stochastic system relaxes towards the equilibrium order state, either a crystalline or a polycrystalline. However, when the transition is first-order, the stochastic system relaxes towards a nonequilibrium glassy state provided the thermodynamic limit be taken before the infinite time limit. Notice the reversion in the order of the limits when compared with the equilibrium case in which the thermodynamic limit is taken after the infinite time limit.

\section{The Potts model}

In the $q$-state Potts model [23] each site of a regular lattice assumes one out of $q$ different possible states, or colour. If two nearest neighbour sites have the same colour their energy contribution is zero, otherwise they contribute by an amount $\epsilon>0$ to the system energy:

$$
E=\sum_{(i j)} \epsilon\left(1-\delta_{\eta_{i} \eta_{j}}\right)
$$

where $\eta_{i}=1,2, \ldots q$ and the sum is over the nearest neighbor sites. There are $q$ degenerate ground states, with zero energy, each one corresponding to having all sites the same colour. We stress that the energy is proportional to the length $\ell$ of the line separating regions of distinct colours (line of defects), 
$E=\epsilon \ell$. We distinguish the states according to the value of $\ell$. If $\ell=0$, there is no defect and the state is crystalline. If $\ell>0$ but negligible, the state is polycrystalline, otherwise, if $\ell$ is not neglible the state is a noncrystalline state which we call a glassy state. By negligible we mean that the density of defects $\rho_{\mathrm{x}}=\ell / N$ vanishes in the thermodynamic limit.

The sudden cooling process is performed by simulating the Potts model at zero temperature according to the Metropolis dynamics [24] starting with a random and uncorrelated configuration to mimic the infinite temperature state. We start by using Periodic Boundary Conditions (PBC). For long times, the system is expected to relax towards the state of minimum energy, $E=0$, where all sites have the same colour. However, this may not happen and the system may be trapped in a noncrystalline configuration, similar to Mondrian or van Doesburg paintings, with many local traps with minimum energy in the shape of a "T" separating different domains. This behavior is well known [25, 26] and is not found when Fixed Boundary Conditions (FBC) are imposed to the lattice, i.e., when one predetermined arbitrary colour is assigned to the sites beloging to the lattice boundary. With this prescription the system is forced to relax to the crystalline state and, after a slow relaxation which occur for an intermediate time regime, the system eventually enters into a fast relaxation stage by which all sites finally assume the same colour of the boundaries.

In fig. 11 we have plotted the density of defects $\rho_{\mathrm{x}}$ against $1 / \sqrt{t}$ obtained from simulations of the 7-state Potts model with both PBC and FBC on a square lattice with $N=L \times L$ sites. Up to an intermediate time regime all curves display the same linear behavior, showing that the density of defects decays like $1 / \sqrt{t}$ [27] independently of both the boundary conditions and the lattice size. At long times the behavior is distinct for each boundary condition. When PBC are used, the system reaches configurations with a nonzero density of defects $\rho_{\times}$. When FBC are used, a fast (exponential) relaxation enters into action at a characteristic time, the system eventually reaches the ground state and $\rho_{\times}$vanishes, as shown in fig. 2. The characteristic time increases with $L$ (inset of the figure). Extrapolation to the thermodynamic limit implies its divergence and the disappearance of the final relaxation towards the ground state. According to fig. 1, the curves corresponding to PBC and FBC approach each other and are expected to become identical in this limit. The extrapolation of the linear region in fig. 1 gives a nonzero value of the density of defects $\rho_{x}^{*}$ when $t \rightarrow \infty$. This nonzero residual density of defects shows that the defects are not negligible in the thermodynamic limit 


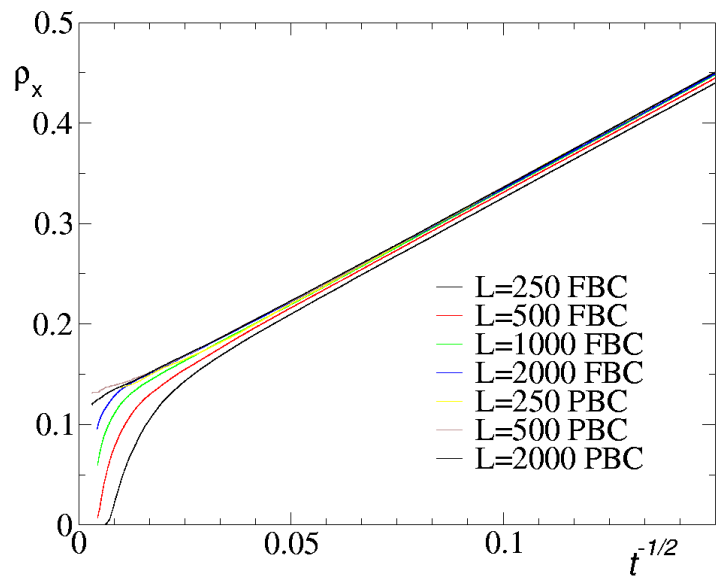

Figure 1: Density of defects $\rho_{\mathrm{x}}$ versus $1 / \sqrt{t}$ at large times, for the 7 -state Potts model on a square lattice for several linear sizes $L$ and different boundary conditions (PBC or FBC).

and we are faced with a glassy states.

We have investigated the density of defects for several values of $q$, as shown in fig. 3, and found that whenever $q>q_{c}=4$ the residual density of defects $\rho_{\mathrm{x}}^{*}$ is nonzero. In the special case of $q=q_{c}$, there are logarithmic corrections in the time decay and our results for $q=4$ suggest the behavior $\rho_{\mathrm{x}} \approx(\ln t / t)^{1 / 2}$. For different values of $q$, the residual densities of defects resulting from our analysis are reported in the inset of fig. 3 .

On the basis of the results obtained, we can draw the following conclusions: $i$ ) for $q=2$ the system relaxes towards a pure crystal (no defects); ii) for $q=3,4$ the system forms a polycrystal (negligible number of defects); iii) for $q>4$ the system relaxes towards a glassy state (nonzero density of defects).

Existence of low temperature noncrystalline states was predicted on a general basis by Lifshitz 28 who argued that a $d$-dimensional system quenched below its critical point does not necessarily equilibrate into a pure state, in the presence of more than $d+1$ ordered ground states. The competition among different possible ordering processes would drive the system away from crystallization, although nothing is given to know about the length of the lines of defects. An interesting investigation on the formation of ordered vs disorered structures in the quench of some two dimensional lattice gas 


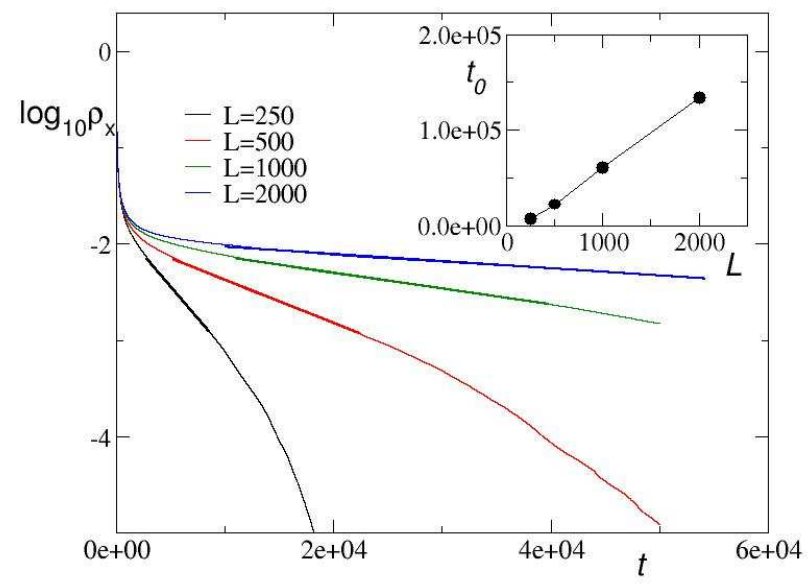

Figure 2: Late stage relaxation to the ground state energy for the Potts model with $q=7$ and FBC. An exponential regime can be detected (thick dashed lines) whose characteristic time $t_{0}$ increases linearly with the lattice size (inset).

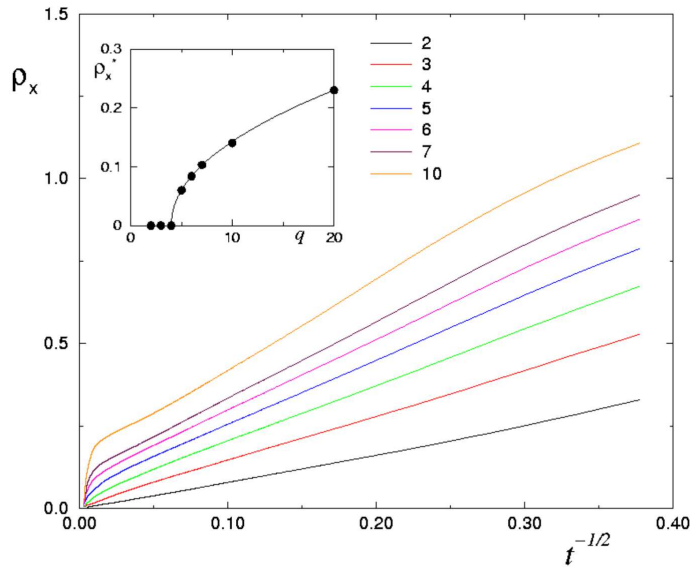

Figure 3: Density of defects $\rho_{\mathrm{x}}$ versus $1 / \sqrt{t}$ for the $q$-state Potts model with $q=2$ up to $q=10$ on a square lattice of linear size $L=10^{3}$ with FBC. The inset shows the residual density of defects $\rho_{\mathrm{x}}^{*}$ versus $q$, including $q=20$. The continuous line is a fitting of the type $\rho_{\mathrm{x}}^{*}=A(q-4)^{\beta}$. The best fitting gives $A=0.060$ and $\beta=0.49$. 
models can be found in [29].

\section{The exclusion models}

In exclusion models, adsorbed particles diffuse over the sites of a regular lattice in such a way that each adsorbed particle excludes the sites of a certain neighborhood from being occupied by other particles. The particles do not interact except through excluded volume repulsion.

In order to give a quantitative definition of a glassy state it can be considered tha case in which such a system is placed in contact with a particle reservoir. The energy of the system will be $-\mu n$ where $\mu>0$ is the chemical potential and $n$ is the number of adsorbed particles. The ground state is that of the closest packing because the number of deposited particles is maximum. Here we set the zero of energy as that of the ground state so that the energy is $E=\mu\left(n_{\mathrm{cp}}-n\right)$ where $n_{\mathrm{cp}}$ is the number of particles in the close-packed state. With this definition, the energy is proportional to the number of vacancies $n_{\mathrm{x}}=n_{\mathrm{cp}}-n$. Again we may define a glassy state as being the state in which the density of vacancies $\rho_{\mathrm{x}}=n_{\mathrm{x}} / N$ is nonzero in the thermodynamic limit. Otherwise the state is either crystalline or polycrystalline.

Sudden cooling process at zero temperature in these systems has been investigated in the literature (a recent review can be found in [17]). It can be performed by simulating the model according to the Metropolis dynamics starting with an empty lattice. According to this dynamics particles either diffuse or are adsorbed. In the absence of diffusion a jamming state would be reached, in which all lattice sites would be blocked [16, 17]. However diffusion of adsorbed particles over the lattice allows for deposition of other particles whenever a site previously blocked is made free. In principle, a close-packed state may eventually be reached in which particles are arranged into a regular periodic pattern.

We have considered exclusion models on a square lattice with different number of ground states. The first model studied ( $N_{1}$ model) has two ground states and is defined by imposing the exclusion of the nearest-neighbour sites. This corresponds to a system of hard squares, each one having an area equivalent to 2 unit cells and thus covering 2 sites of the lattice. The close-packed state in this case consists of a checkerboard-like arangements of particles and can occur on two different sub-lattices. Models with a different number of ground states can be designed in a similar way, by the exclusion 


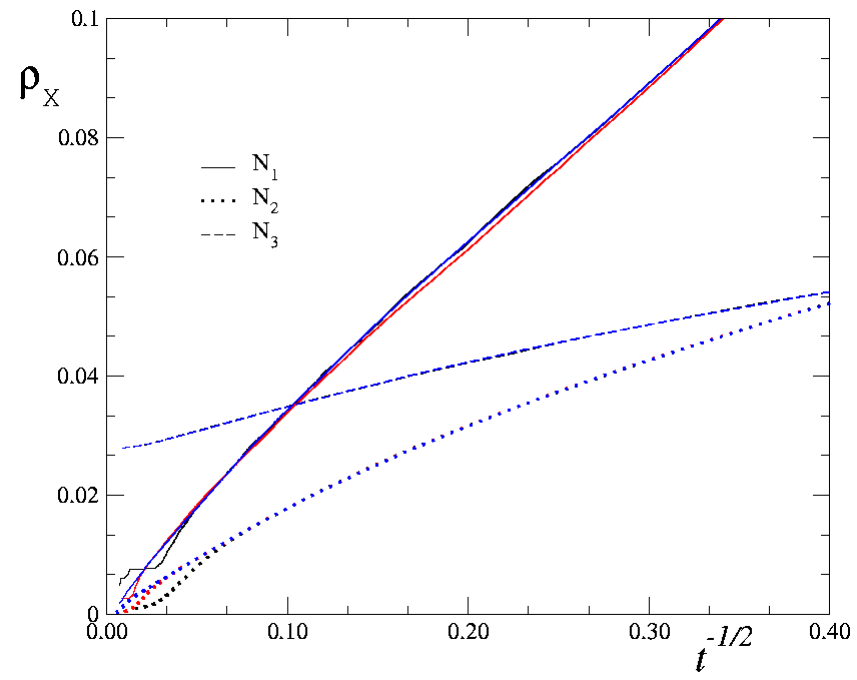

Figure 4: Density of vacancies $\rho_{\mathrm{x}}$ versus $1 / \sqrt{t}$ for the exclusion models $N_{1}$, $N_{2}$ and $N_{3}$ on a square lattice. For each model, the linear size of the lattice are $L=50,100$ and 200 .

of a suitable set of neighbours.

It is known [19] that for the $N_{1}$ model, the deposition-diffusion process leads to ordering into one of the two ground states. For determining the behavior of models with a different number of ground states we have investigated numerically the models with exclusion of both first and second neighbors, $N_{2}$ [20, 21] and of third neighbors as well, $N_{3}$ [22]. The $N_{2}$ model corresponds to a system of hard plane objects, each one covering 4 sites of the lattice; whereas in $N_{3}$ each hard object covers 5 sites. In analogy with the case of the Potts model we have plotted the density of vacancies $\rho_{\mathrm{x}}$ versus $1 / \sqrt{t}$.

Fig. 4 shows the decay of $\rho_{\mathrm{x}}$ for the $N_{3}$ model. Extrapolation to $L=\infty$ allows to conclude that in this case $\rho_{\mathrm{x}} \rightarrow \rho_{\mathrm{x}}^{*}>0$ when $t \rightarrow \infty$. On the contrary, simulations of the $N_{2}$ model reported also in fig. 4 show that in this case $\rho_{\mathrm{x}} \rightarrow 0$ when $t \rightarrow \infty$, although as $\rho_{\mathrm{x}} \approx(\ln t / t)^{1 / 2}$. By considering the number $q$ of sites covered per hard plane object in each model we are thus lead to the same conclusions as for the Potts model: $i$ ) in the $N_{1}$ model, $q=2$ and the system relaxes towards a pure crystal (no vacancies); ii) in the $\mathrm{N}_{2}$ model, $q=4$ and the system forms a polycrystal (negligible number 
of vacancies); iii) in the $N_{3}$ model, $q=5$ and the system relaxes towards a glassy state (nonzero density of vacancies).

\section{Discussion}

These conclusions show that the ability of these systems to form glassy states depends on the number of equivalent crystalline states as a consequence of the competition among different ordered domains. The critical number $q_{c}$ of equivalent crystalline states above which the glassy state emerges is found to be $q_{c}=4$ both for the Potts and the exclusion models on a square lattice.

We have also perfomed numerical simulations of the Potts model on a triangular lattice for values of $q$ ranging from $q=2$ up to $q=12$ and found that the density of defects vanishes in the infinite time limit. These results are consistent with the results obtained by Sahni et al 30] in which domains grow without limits in the Potts model on a triangular lattice but are pinned on a square lattice.

The results obtained on the square lattice suggest that the observed glassy states might be related to the presence of a first-order transition. In fact, the equilibrium two-dimensional Potts model suffers a phase transition from a disordered state, at high temperatures, to an ordered state with $q$ coexisting phases, at low temperatures, which is continuous for $q \leq 4$, and first-order for $q>4$ [23]. Analogous features have been observed in the exclusion models studied here: In equilibrium, $N_{1}$ and $N_{2}$ models display a secondorder transition whereas the $N_{3}$ model show a first-order transition [18, 31, 32 .

\section{Conclusion}

The results presented in this letter show that systems with translational invariance and short range interactions may be driven away from crystallization by the competition among a number of coexisting ordered states. For the models considered here, i. e. the Potts model and the exclusion models on the square lattice, the number of states above which ordering is destroyed corresponds to the appearance of a first-order transition in the equilibrium diagram of the systems and is the same for the two models.

These results are obtained by reversing the usual order in which limits are 
taken when doing equilibrium statistical mechanics, i. e. the limit $L \rightarrow \infty$ is taken before $t \rightarrow \infty$. Within this approach, our simulations indicates that the characteristic time for the system to relax to the ground states may grow faster than the system size, in the sense that thermodynamic equilibrium is never attained 33, 34.

\section{References}

[1] Lipowski A., J. Phys. A 30 (1997), 7365.

[2] Swift M. R., Bokil H., Travasso R. D. M. Bray A. J., Phys. Rev. B 62 (2000), 11494.

[3] Franz, S, Mezard M., Ricci-Tersenghi F., Weigt M. Zecchina R., Europhys. Lett. 55 (2001), 465.

[4] Biroli G. Mezard M., Phys. Rev. Lett. 88 (2002), 25501.

[5] Weigt M. Hartmann A. K., cond-mat/0210054.

[6] Pica Ciamarra M., Tarzia, M., de Candia A. Coniglio A., cond-mat/0210144.

[7] A. P. Young Ed., Spin Glasses and Random Fields World Scientific, Singapore (1998).

[8] Santen L. Krauth W., Nature 405 (2000), 550.

[9] Angelani L., Parisi G., Ruocco G. Viliani G., Phys. Rev. Lett. 87 (2001), 5502.

[10] Kob W. Andersen H. C., Phys. Rev. E 48 (1993), 4364.

[11] Davison L., Sherrington D., Garahan J. P. Buhot A., J. Phys. A 34 (2001), 5147.

[12] Griffiths R. B., J. Math. Phys.5 (1964), 1215.

[13] Yeomans J. M., Statistical Mechanics of Phase Transitions, Oxford University Press, New York, (1992). 
[14] Gleiser P. M., Tamarit F. A. Cannas S. A., Physica D 168-169 (2002), 73.

[15] Antoni M. Ruffo S., Phys. Rev. E 52 (1995), 2361.

[16] Evans J. W., Rev. Mod. Phys. 65 (1993), 1281.

[17] Privman V., Colloids Surf. A 165 (2000), 231.

[18] Runnels L.K., Phase Transitions and Critical Phenomena C. Domb M. S. Green Eds., Vol. 2 Academic Press, London (1972), p. 305.

[19] Wang J. S., Nielaba P. Privman V., Physica A 199 (1993), 527.

[20] Wang J. S., Nielaba P. Privman V., Mod. Phys. Lett. 7 (1993), 189.

[21] Eisenberg E. Baram A., Europhys. Lett. 44 (1998), 168.

[22] Eisenberg E. Baram A., J. Phys. A 33 (2000), 1729.

[23] Wu F. Y., Rev. Mod. Phys. 54 (1982), 235.

[24] Metropolis N., Rosenbluth A. W., Rosenbluth M. N., Teller A. H. Teller E., J. Chem. Phys. 21 (1953), 1087.

[25] Anderson, M. P. Grest G. S. Srolovitz D. J., Phil. Mag. B 59 (1989), 293, and refs. therein.

[26] Derrida B., de Oliveira P. M. C. Stauffer D., Physica A 224 (1996), 604.

[27] Bray A. J., Adv. Phys. 43 (1994), 357.

[28] Lifshitz I. M. Zh. Eksp. Teor. Fiz. 42 (1962), 1354, transl. Sov. Phys. JETP 15 (1962), 939.

[29] Sadiq A. Binder K., J. Stat. Phys. 35 (1984), 517.

[30] Sahni P. S., Srolovitz D. J., Grest G. S. Anderson M. P. Safran S. A., Phys. Rev. B 28 (1983), 2705.

[31] Orban J. Van Belle J. D., J. Phys. A 15 (1982), L501.

[32] Domany E., Schick M. Walker J. S., Phys. Rev. Lett. 38 (1977), 1148. 
[33] Schulman L. S., Finite Size Scaling and Numerical Simulations of Statistical Systems V. Privman Ed., World Scientific, Singapore (1990), p. 489 .

[34] Biroli G. and Kurchan J., Physical Review E 64 (2001), 16101. 